\title{
A Comparison Study Between the Downlink Packet Scheduling Algorithms in LTE Networks
}

\author{
Mohammed A. Suliman Dr. Saad Ahmed Ayoob \\ M.Sc. Student \\ Lecturer \\ Electrical Eng. Dept., College of Engineering, Univ. of Mosul
}

\begin{abstract}
LTE networks are the latest generation of wireless networks based on IP architecture; it is standardized by the 3GPP in its Rel.8. LTE networks are implemented practically to access internet with very high data rates with using various multimedia services. This paper is based on five types of downlink packet scheduling algorithms in LTE networks, It examines the impact of throughput, the fairness and the spectral efficiency of each scheduling algorithms when the speed of UE (User Equipment) and the number of UEs are changed. A comparison between the scheduling algorithms has been done using Vienna LTE SYSTEM LEVEL Simulator.
\end{abstract}

Key words: LTE, scheduling, algorithms, SYSTEM LEVEL.

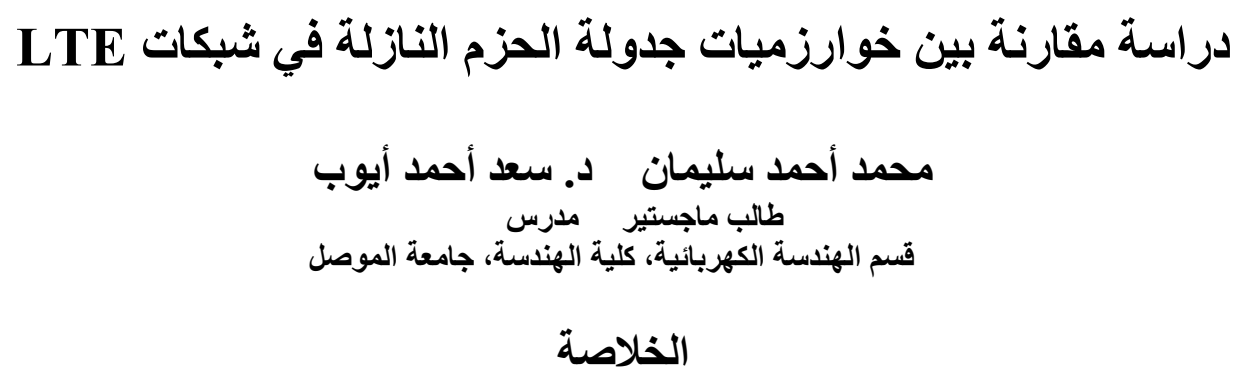

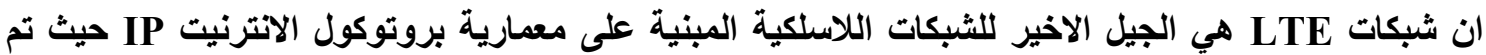

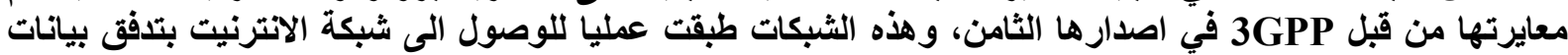

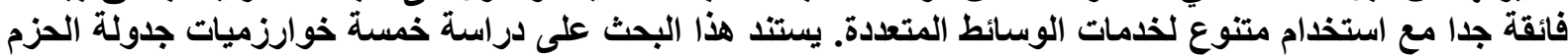

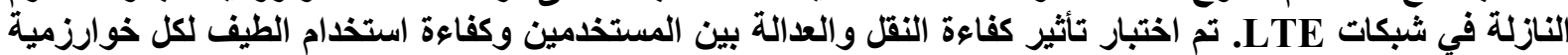

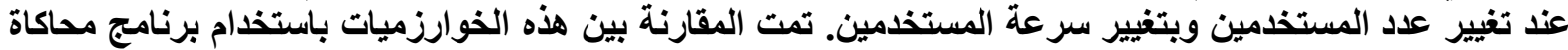

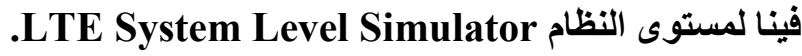




\section{Introduction}

The rapid growing demands for network services such as VoIP, web browsing, video telephony and video streaming, especially real time traffics, impose strong challenges on the design of wireless networks [1]. 3rd Generation Partnership Project (3GPP) is the organization which introduced the LTE specifications all-IP architectures for the core networks and the radio access. It includes large capacity and high speed of mobile telephone networks. LTE system is based on a flat architecture called Service Architecture Evolution as shown in Fig. 1 [2-4].

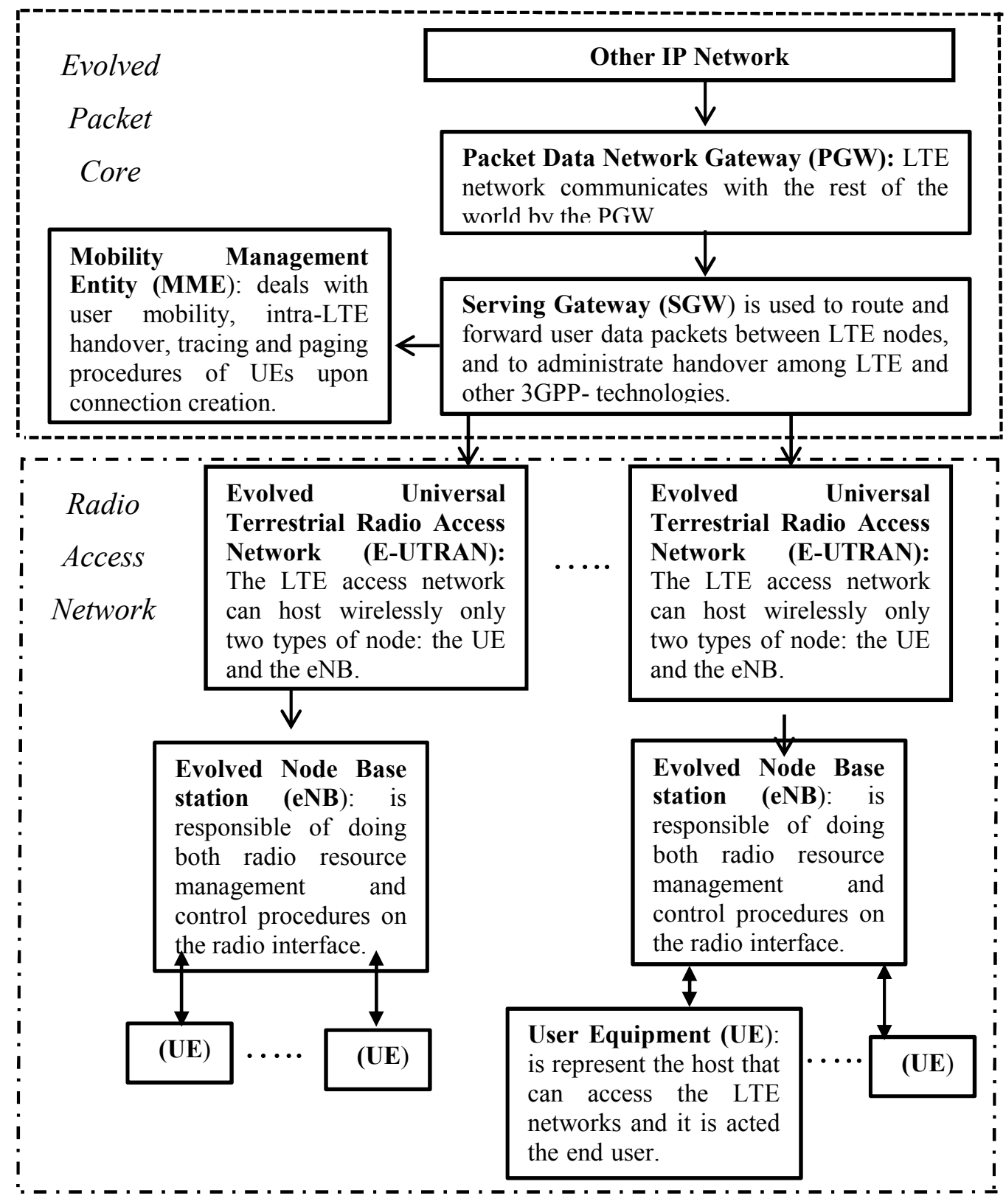

Fig. 1: Flow chart of LTE network architecture. 
Multi-user scheduling is one of the main features in LTE systems because it is responsible of distributing available resources among active users to provide the required QoS [2]. LTE is based on Orthogonal Frequency Division Multiple Access (OFDMA) at the downlink and single-carrier frequency division multiple access (SC-FDMA) at the uplink. The minimum resource unit that the MAC scheduler allocates to a UE in OFDMA is a Resource Block (RB) in each transmission time interval (TTI) [5-8].

In the literature, B. Liu, H. Tian, and Lingling $\mathrm{Xu}$ displayed the performance of five LTE Downlink Packet Scheduling Algorithms in terms of traffic flow throughput, packet loss ratio (PLR), packet delay, fairness index, and cell spectral efficiency [1]. Capozzi F., Boggia G., Piro G., Grieco L.A. and Camarda P. are offered an overview on the key issues that ascend in the design of a resource allocation algorithm for LTE networks [2]. D. Zhou, W. Song, N. Baldo and M. Miozzo introduced three LTE Downlink MAC Schedulers in a Vehicular Environment and examined the different TCP throughput achieved with frequency and time domains [4]. R. Kwan, C. Leung and J. Zhang discussed how to allocate resources to multiple users on the downlink of LTE and presented the performance of a scheduler with fairness among users [5]. H. Al-Jaradat and K. Sandrasegaran showed the performance of three LTE Downlink Packet Scheduling Algorithms in terms of packet throughput, packetloss ratio, packet latency, fairness index and total cell spectral efficiency [7]. S. A. Alqahtani and M. Alhassany studied the performance of six LTE downlink packet scheduling algorithms in terms throughput and fairness index with speed and SNR by using link level simulator [8]. S. A. Al-Qahtani and M. Al-Hassany are suggested a novel scheduling algorithm for LTE networks and compared its performance with the performances of both the Best-CQI and RR Uplink schedulers [9]. M. H. Habaebi, J. Chebil, A. G. Al-Sakkaf and T. H. Dahawi are studied the performance of three types of scheduling algorithms [10]. F. Bendaoud, M. Abdennebi and F. Didi are examined several scheduling algorithms which proposed for LTE for uplink and downlink [11].

This paper deals with five types of downlink packet scheduling algorithms in LTE networks. Max throughput, Round Robin, Proportional Fairness, Resource fair and Best CQI scheduling algorithms. It is important to compare these scheduling algorithms to show the strengths and weaknesses points of these scheduling algorithms. It includes the results of the impact of the significant criterions of networks (the throughput, spectral efficiency and the fairness of each scheduling algorithm). Also it has been examined the results of each one when the speed of UEs have been changed from 5 to $320 \mathrm{Km} / \mathrm{h}$ and also the number of UEs have been changed from 10 to 100 UEs/eNB. The analysis has been done using Vienna LTE SYSTEM Level Simulator v1.8 r1375 [12] which it is used in most papers as presented [2, 3, $9,10]$.

The remain of this paper will be as follows. Section II offers in detail of the different types of scheduling algorithms. Simulations and results are introduced in Section III. Finally, Section VI refers to the Conclusion and Recommendations of the paper. 


\section{Types of scheduling algorithms \\ A- Best Channel Quality Indicator (BCQI) scheduler:}

BCQI algorithm is allocated a RBs to the UE with the best radio link conditions where the UE that has best values of CQI and will capture of resource in the cell. This scheduling is increased the cell throughput without taking the fairness into account where the UEs in celledge are improbable to be scheduled $[9,10]$. The scheduler selects the user with a maximal priority metric, which is defined as [3]:

$$
j=\max _{i=1 \text { toN }}(\operatorname{Ri}(t))
$$

Where, $j$ is the selected user, $\operatorname{Ri}(t)$ is the realizable data rate of UE $i$ at time $t, \mathrm{~N}$ is the total number of active users and $i$ is the user index.

\section{B- Round Robin (RR) Scheduler}

In this scheduling algorithm are assigned the RBs for the UEs one after another; therefore, it is ensured a fairness for all users, thus every user will got same amount of RBs without taking the CQI into account. The UEs are served on the basis ( first come first serve ). accordingly, it don't ensured cell throughput. It is easily to realized [9 - 11]. Transmission service will satisfy the following equation [3]:

$$
j=\max _{i=1 \text { toN }}(Q i(t))
$$

Where, $\mathrm{j}$ is the selected user, $\mathrm{N}$ is the total number of active users, $Q i(t)$ is the user queue length and $i$ is the user index.

\section{C- The Proportional Fair (PF) scheduler:}

This scheduling algorithm can balance between cell throughput and fairness, it allocates RBs to UEs with taking in to account the CQI in the first time slot while providing an equal throughput to each UE in the second time slot in each TTI $[4,10,11]$.The scheduler selects the user with a maximal priority metric, defined as [3]:

$$
j=\arg \frac{\max _{i \leq i \leq N}(D i(t))}{\operatorname{Ri}(t-1)}
$$

Where $i$ is the user index, $j$ is the selected user, $N$ is the total number of users, $D i(t)$ is the current supportable data rate by the channel, and $R i(t-1)$ is the average data rate by this user. With this scheme, the selected user must have a good channel or high data rate $D i(t)$, to keep system throughput high.

\section{D- Resource Fair (RF) Scheduler:}

The fairness with respect to the number of RBs will be actualized, where it provides an equal amount of resources for all users. This can be realized as [8]:

$$
T_{i}=\frac{R}{N}
$$


Where:

$T_{i}$ is the number of RBs that allocates to user $i, R$ is the total number of RBs and $N$ is the total number of UEs that scheduled at each TTI; therefore, each UE will get same number of RBs to guarantee fairness.

\section{E- Maximum Throughput (MT) scheduler:}

The MT scheduler assigns RBs to UEs which have the best CQI. Active users are ordered according to their CQI and organized in the downward order in each TTI. The MT allocates RBs to the user that have the maximum likely data rate firstly and then assigns in a round robin technique according to their CQIs. The target of this scheduler is to maximize the total throughput of an eNB [4].

The fairness of how equally a resource can be estimated with a given throughput can be suggested over $\mathrm{N}$ users using the following equation [8]:

$$
\mathrm{F}\left(R_{1}, R_{2}, \ldots, R_{\mathrm{i}}\right)=\frac{\left(\sum_{\mathrm{i}=1}^{\mathrm{N}} R_{i}\right)^{2}}{N \sum_{i=1}^{N} R_{i}{ }^{2}}
$$

Where: $R_{i} \geq 0$

$F\left(R_{)}\right)=$Fairness index

$R_{i}=$ The average throughput of user $i$.

$\mathrm{N}=$ No. of contending users.

The efficiency coefficient has range from (0) to (1) where it is used to compare the scheduling algorithms. It is described the ratio of a mean normalized UEs throughput to the maximum normalized UEs throughput. The maximum value of the efficiency coefficient is one this mean that all UEs in any place of a cell have the same amount of throughput, as appear in the equation below [3]:

$$
\text { Efficiency coefficient }(\eta)=\frac{M_{t h r}}{S_{\max }}
$$

Where $M_{t h r}$ is the mean of the normalized UEs throughput.

$S_{\max }$ is the maximum normalized UEs throughput.

The spectral efficiency is the number of the bits transmitted per Hertz. It has the ability to maintain high throughput of the cell where it represents bit/sec./Hz. Each value of spectral efficiency corresponded to specific value of CQI; therefore, the spectral efficiency increase when the CQI increased and then the throughput of the cell will be increased. $[10,11]$.

\section{Simulation results}

The simulation results of five scheduling algorithms are compared through the following scenarios where it is consists of two parts, the first is focused on increasing number of UEs and the second is dedicated on rising a speed of UEs. It has been done using Vienna LTE System Level Simulator v1.8 r1375. 
Part I: Increasing number of UEs with fixed speed of UEs at $5 \mathrm{~km} / \mathrm{h}$

Scenario 1: This scenario arranged with the following parameters: $2 \times 2$ closed loop Spatial Multiplexing (CLSM), 20 $\mathrm{MHz}$ bandwidth, Winner II channel, 30 UEs/cell, 10UE/sector, Tri-sector, 5 $\mathrm{km} / \mathrm{h}$ user speed, $0.25 \mathrm{~km}$ Cell radius, 100 TTI simulation time. Full buffer traffic model, Seven base stations each contains three sectors (eNB). Where the region of interest (ROI) contains seven base stations each one contains three eNBs as displayed in Fig. 2. The blue dots represent the UEs and the red dots represent the base stations (BS).

The empirical cumulative distribution function (ECDF) with the average

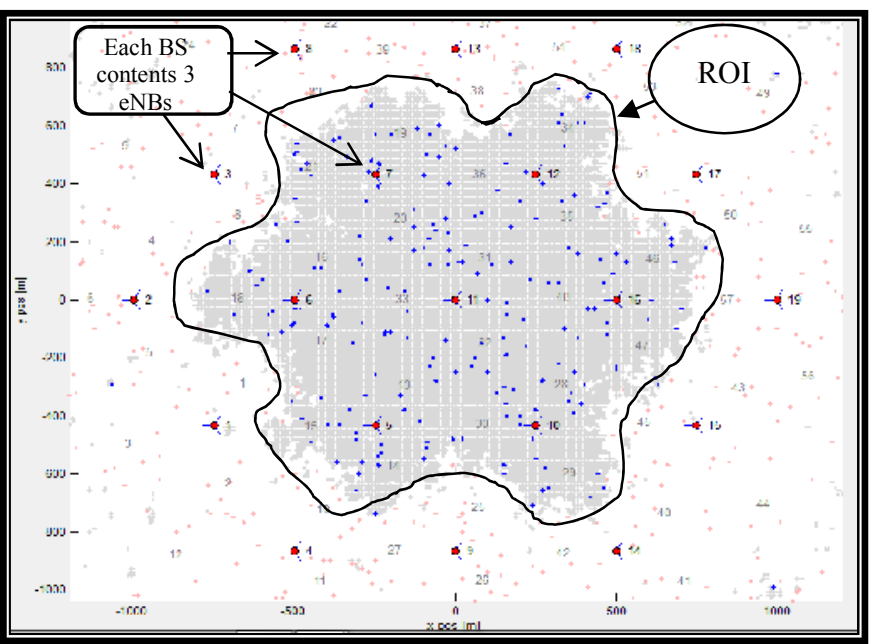

Fig. 2: Seven Base station with10UEs/sector. throughput of UEs at 10UEs/sector is illustrated by Fig. 3.. The black dots represent the mean throughput of UEs. The Best CQI Algorithm has the larger value among the other scheduling algorithms but it has about $60 \%$ of UEs have Zero throughput and the others have mean throughput about $12 \mathrm{Mbps}$. Therefore, it does not satisfy the required fairness. The resource fair scheduling algorithm is the best one in term of fairness however the mean throughput for UEs is less than $6 \mathrm{Mbps}$ and the other scheduling algorithms are between them.

The mean and peak throughputs together with the actual throughput of UEs at the edge of cell at 10UEs/sector and $5 \mathrm{~km} / \mathrm{h}$ User speed can be clarified by Fig. 4 shows that the best CQI algorithm has the larger values of mean throughput and peak throughput of UEs among the other algorithms, on the other hand it don't have any throughput of UEs at the edge of the cell, this mean that all the RBs are allocated to UEs whose near BS.

It is worth to mentioned, that the RR, RF and PF algorithms are allocated the RBs to UEs at the edge of the cell because of these algorithms verify the fairness among UEs, contrariwise the BCQI and MT don't assign any RB to UEs at the edge of the cell because of these algorithms don't prove the fairness among UEs.

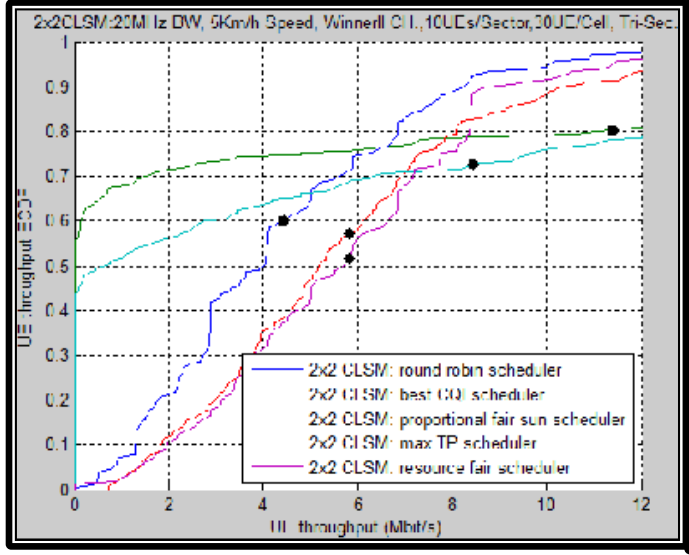

Fig. 3: ECDF with throughput.

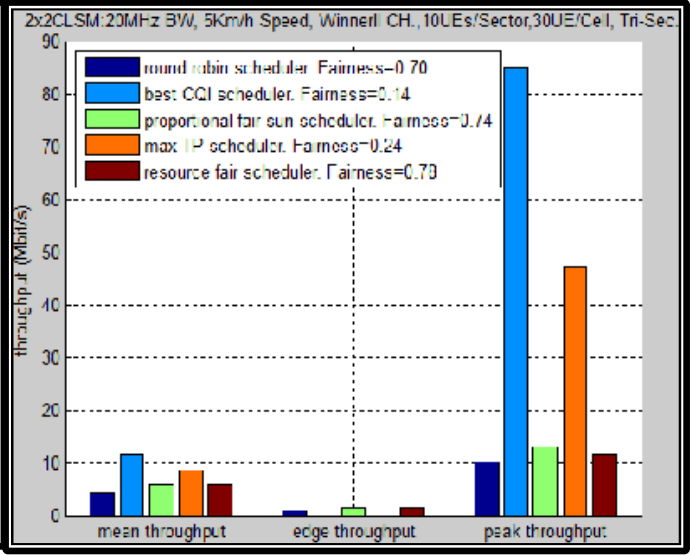

Fig. 4: Throughput of UEs. 
Scenario 2: The parameters of this scenario similar to that of scenario 1 except the number of UEs which become $150 \mathrm{UEs} /$ cell and 50UE/sector at the same user speed. Fig. 5 illustrates the ECDF with the average throughput of UEs. The black dots represent the mean throughput of UEs. The Best CQI Algorithm has higher value than the other scheduling algorithms with about $85 \%$ of UEs have zero throughput while the others have mean throughput about $3 \mathrm{Mbps}$. Therefore, the fairness among UEs is determined as the number of UEs per eNB increases. The resource fair scheduling algorithm is the best fairness which equal 0.78 , in the other hand, the mean throughput for UEs is less than $1.4 \mathrm{Mbps}$ and the other algorithms are between them.

As expected, the throughput of UEs decrease as the number of UEs per cell increases. Fig. 6 illustrates that the Best CQI Algorithm has higher UEs values of mean and peak throughputs among the other algorithms but it has zero throughput at the edge of cell. RR, RF and PF algorithms are used to allocate the resources to the UEs at the edge of cell, PF is the best since it can allocate larger amount of resources to the UEs at the edge of the cell.

Scenario 3: The number of UEs/cell of this scenario is 300 with 100UE/sector, the remaining parameters are as mentioned in the scenario 1. Fig.7 shows a heavy density of UEs in the ROI. The BSs doesn't allocate any resources for the UEs out of ROI. It is worth to mention that the number of the outside UEs are increased with increasing the UEs per eNB.

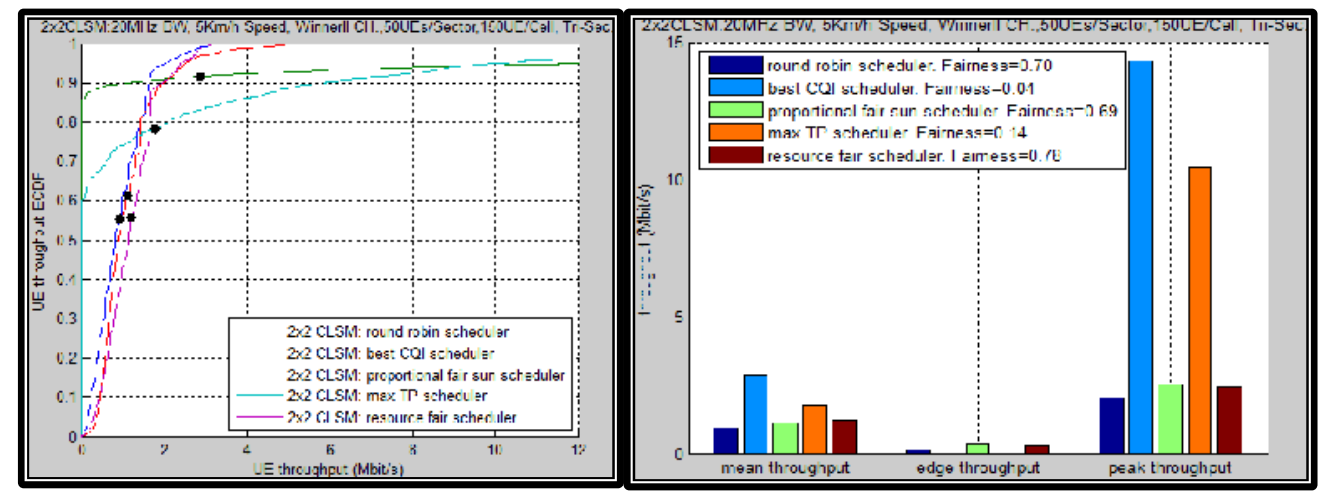

Fig. 5: ECDF with throughput.

Fig. 6: Throughput of UEs.

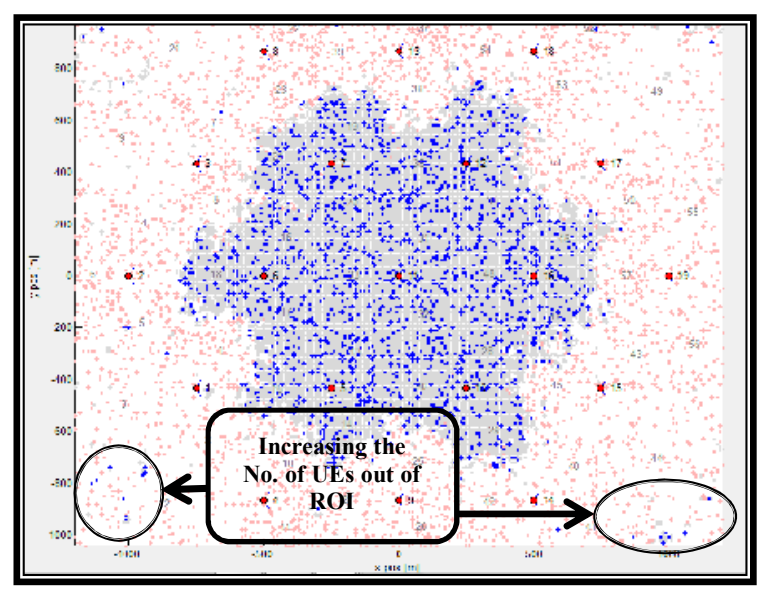

Fig. 7: ROI with 100UEs/sector.

The ECDF with the average throughput of Ues is illustrated in Fig. 8. The Best CQI has the larger mean throughput among the others, it has about $90 \%$ of UEs having zero 
throughput while the other UEs have mean throughput of about $1.5 \mathrm{Mbps}$. Therefore, badness is increased in term of the fairness among the UEs when the number of the UEs per eNB is increased. The resource fair scheduling algorithm is the best one in term of the fairness, it has 0.78 of the fairness with mean throughput for the UEs of less than $0.8 \mathrm{Mbps}$, the other scheduling algorithms are between them.

The throughput of the UEs is decreased as the number of the UEs per cell is increased as shown in Fig. 9. Always the Best CQI Algorithm has the larger values of mean and peak throughputs of UEs among the other Algorithms, on the other hand; it has zero throughput of UEs at the edge of cell.

The three algorithms that allocate the resources to the UEs at the edge of cell are round robin, resource fair and proportional fair. It has been noted that the proportional fair has the maximum throughput of UEs at the edge of the cell.

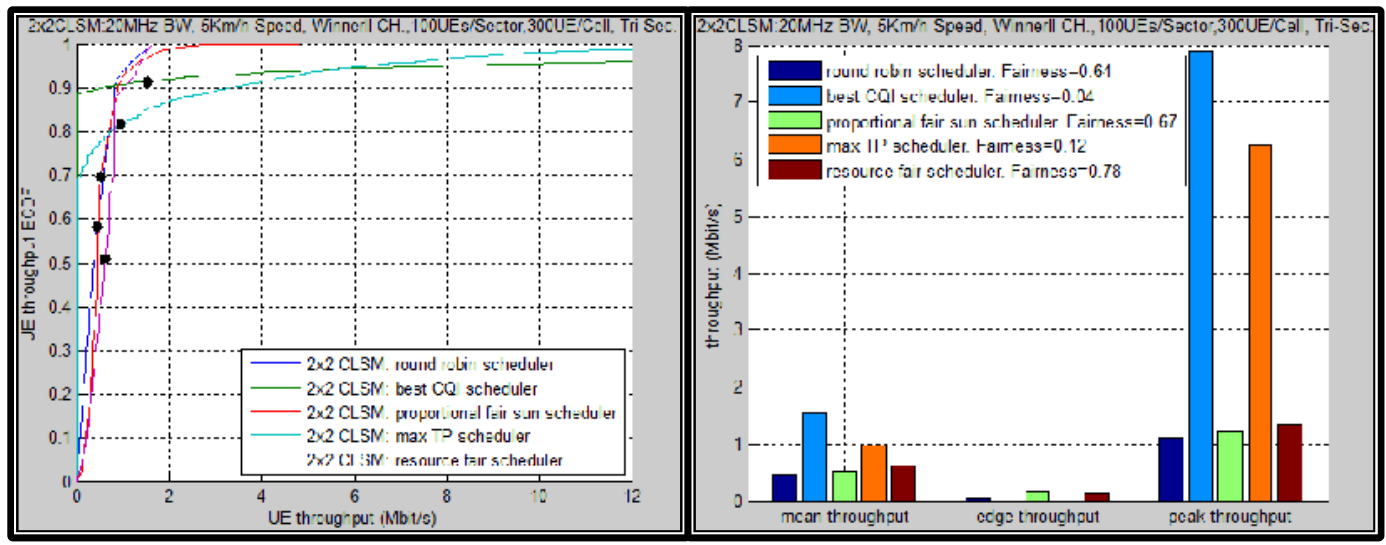

Fig. 8: ECDF with throughput

Fig. 9: Throughput of UEs.

Scenario 4: The number of the UEs is changed from (10-100) UEs/eNB, The average speed of users is $10 \mathrm{~km} / \mathrm{h}$, the remain parameters are as in Scenario 1.

Fig. 10 declared that the mean throughput of UEs for all scheduling algorithms is decreased with increasing the number of UEs per cell. The best CQI has the larger values of mean throughput than the other scheduling Algorithms where it has a mean throughput about $6 \mathrm{Mbps}$ larger than the lower one at $10 \mathrm{UEs} / \mathrm{eNB}$. On the other hand, the mean throughput becomes about $1 \mathrm{Mbps}$ at $100 \mathrm{UEs} / \mathrm{eNB}$.

Fig. 11 shows the fairness against the number of UEs for the five scheduling algorithms as described by eq. 5 where the best CQI and the maximum throughput are decreased with increasing the number of the UEs per cell. The best CQI algorithm has the lower values of the fairness among the other scheduling algorithms when it reaches zero (with increasing the number of the UEs). The other algorithms RR, RF and PF don't affect significantly with increasing number of the UEs. It is noted that the best algorithm is RF in term of the fairness when increasing no. of the UEs.

The spectral efficiency will not change significantly for all scheduling algorithms as shown in Fig. 12 because of the number of UEs outside ROI will increase with increase the total number of UEs as shown in the Fig. 7, additionally, the spectral efficiency depends on the channel conditions where it don't effect with increasing the UEs as mentioned [10]. The best CQI achieved higher spectral efficiency than the others from (10-100) UEs/eNB. 


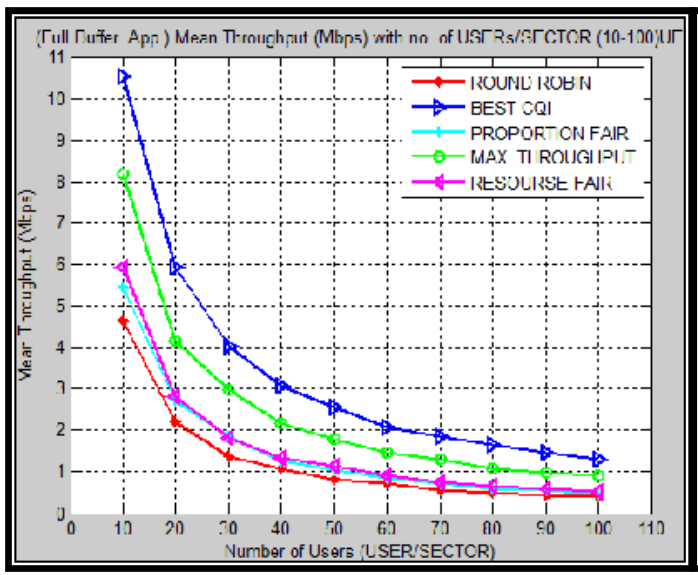

Fig. 10: Throughput vs. no. of UEs.

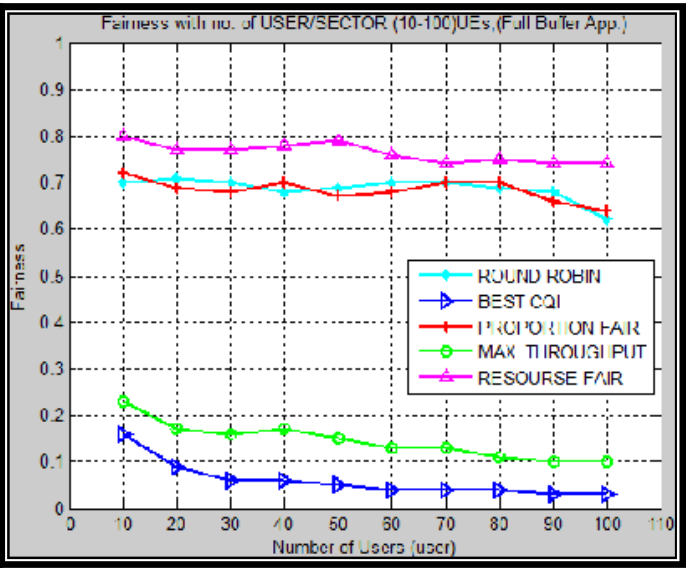

Fig. 11: Fairness vs. no. of UEs.

results of throughput and fairness are validated and compared with the results as $[2,8,10]$. It is showed that BCQI is always realized high throughput, RR is obtained a lower one and PF has throughput in between them, on the other hand, the fairness of the algorithms don't change significantly with increasing number of UEs.

It is obvious from Fig. 13 that the best CQI and the maximum throughput has low efficiency coefficient than the others depending on eq. 6 when the number of UEs changes from (10-100) UEs/eNB. On the other hand, the resource fair achieved higher efficiency than the others when reached of $80 \mathrm{UE} / \mathrm{eNB}$. Round robin achieves higher efficiency coefficient than the others when the number of UE is greater than $80 \mathrm{UEs} / \mathrm{eNB}$.

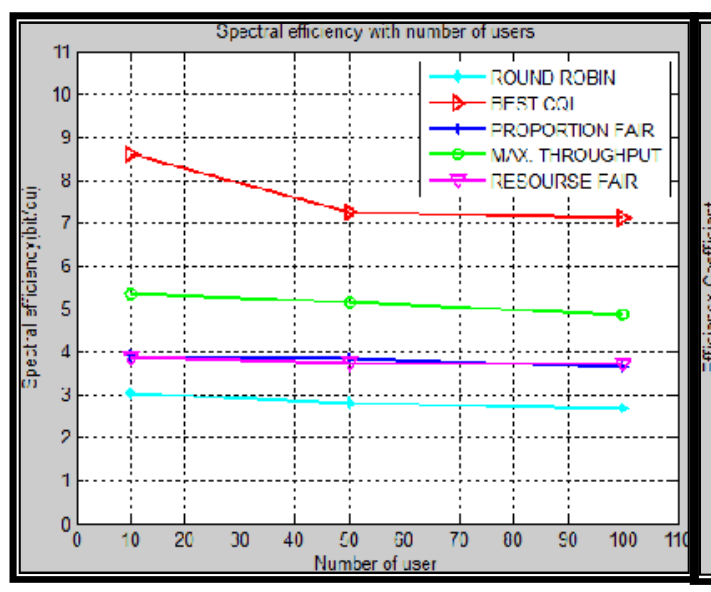

Fig. 12: Spectral efficiency vs. no. of UEs

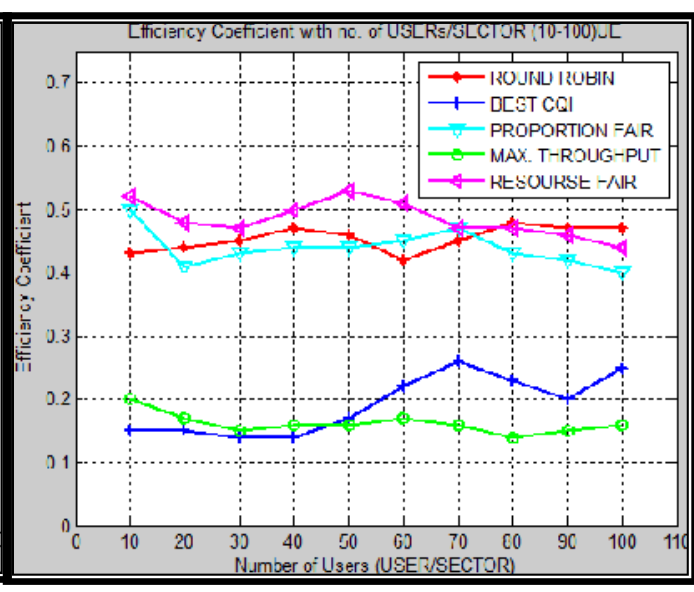

Fig. 13: Efficiency vs. no. of UEs

Part II: Increasing a speed of UEs with fixed number of UEs.

Scenario 5: The effect of a speed of UEs on the throughput is illustrated in this scenario. The parameters are as in scenario 1 with $30 \mathrm{UEs} /$ cell, 10UE/sector at average speed of User is ( 80 and 160$) \mathrm{km} / \mathrm{h}$. Fig. 14 shows the ECDF with the average throughput of UEs at $(80) \mathrm{km} / \mathrm{h}$. The Best CQI also has the larger value among the others which has about $75 \%$ of the UEs having zero throughput while the others have mean throughput about 4.2 Mbps. As 
mentioned in the previous scenarios, the Best CQI increases badness in term of the fairness when the speed of UEs is increased. The round robin is the best one in term of the fairness, it has 0.58 of the fairness while a mean throughput equal to $3.8 \mathrm{Mbps}$, the other scheduling algorithms are between them.

The throughput decreases with increasing average speed of UEs as shown in Fig.15, Best CQI algorithm has the larger values of mean and peak throughputs of the UEs than the other Algorithms. There is no throughput at the edge of cell for all scheduling algorithms except round robin algorithm where it allocates resources to the UEs at the edge of cell when the speed of UEs reaches $80 \mathrm{Km} / \mathrm{h}$.

Clearly, the RR algorithm is worked at excessive speed of users, too, while the other algorithms don't operate at $(160) \mathrm{km} / \mathrm{h}$, as demonstrated in Fig. 16. The mean throughput of $\mathrm{RR}$ algorithm is improved to be the second one after BCQI algorithm. RR is the best to use at excessive speed because of it has the higher value of the fairness and it is the unique algorithm that work at high speed.

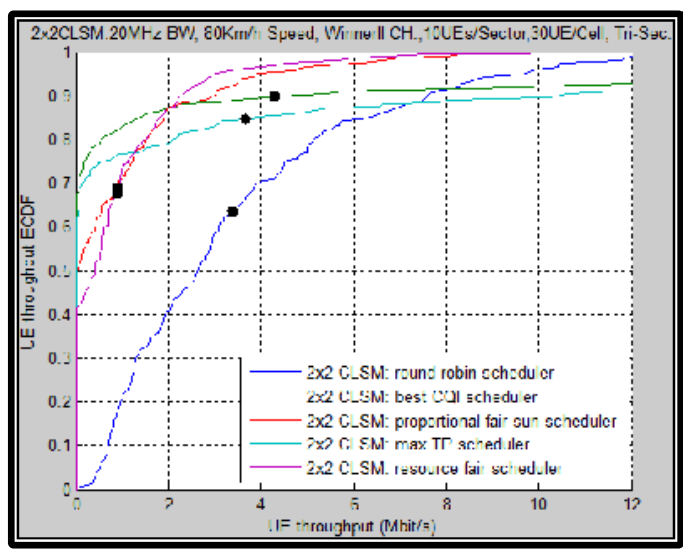

Fig. 14: ECDF with throughput.

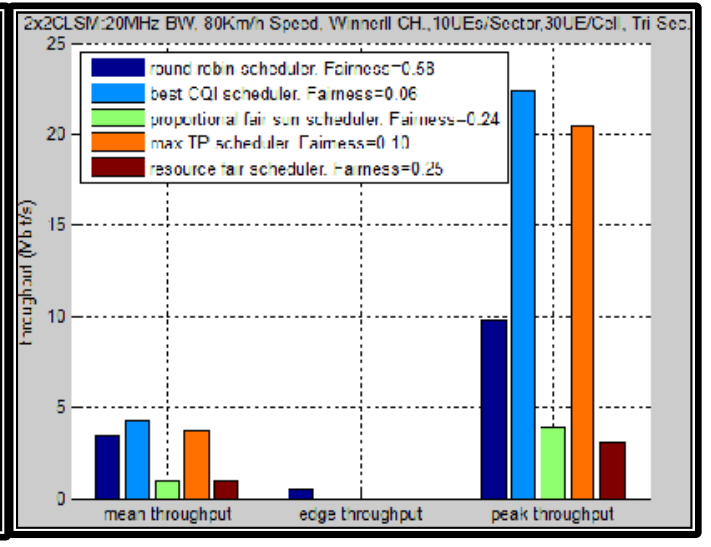

Fig. 15: UEs Throughput at $80 \mathrm{~km} / \mathrm{h}$.

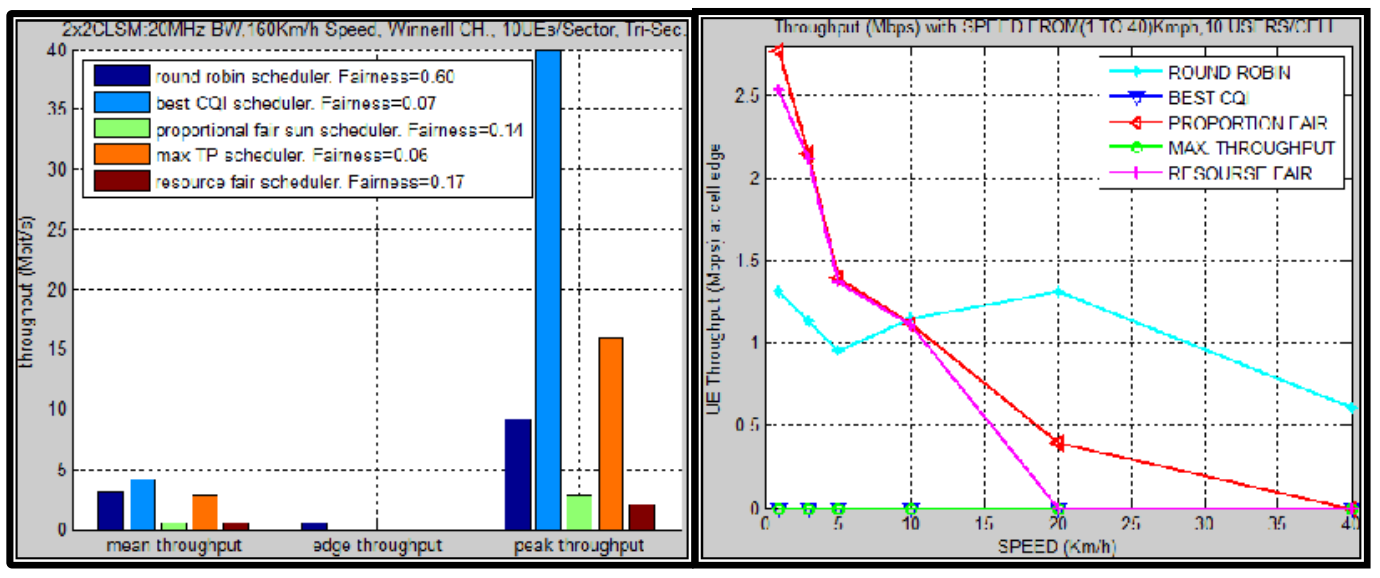

Fig. 16: UEs Throughput at $160 \mathrm{~km} / \mathrm{h}$.

Fig. 17: Throughput with speed.

Scenario 6: It trades with the effect of varying the speed of UEs on throughput of the UEs at the cell edge where it has been increased the speed of UEs at range $(3-40) \mathrm{Km} / \mathrm{h}$. The BCQI and the MT algorithms have zero throughput at the edge of cell. It is found that the proportional fair has larger throughput than the others, when the speed of UEs is $10 \mathrm{Km} / \mathrm{h}$. All 
scheduling algorithms has zero throughput when the speed of UEs is $40 \mathrm{Km} / \mathrm{h}$ except Round robin which has larger throughput than the others given that the speed is greater than 10 $\mathrm{Km} / \mathrm{h}$, it is the unique scheduling algorithm which is succeeded to allocate resources to the UEs at the edge of cell given that the speed is greater than $40 \mathrm{Km} / \mathrm{h}$, see Fig. 17. Therefore, Round robin is suitable for excessive speed usage.

Scenario 7: This scenario is an extension to the previous scenario, the speed of UEs changes from $(5-320 \mathrm{Km} / \mathrm{h})$ at different numbers of the UEs. The Best CQI has the larger values of mean throughput than the others as clarified in the Fig.18, which illustrates that the mean throughput is not changing considerably with increasing the speed of UEs to a value greater than $80 \mathrm{Km} / \mathrm{h}$ for all algorithms.

It is clear that the best CQI scheduling algorithm which is achieved has higher spectral efficiency than the others for the range $(5-320 \mathrm{Km} / \mathrm{h})$ at $150 \mathrm{UEs} /$ cell as shown in Fig. 19. All scheduling algorithms decrease rapidly with increasing the speed of UEs (from $5 \mathrm{Km} / \mathrm{h}$ to $80 \mathrm{Km} / \mathrm{h}$ ). The spectral efficiency is not changed significantly for all scheduling algorithms at $80 \mathrm{Km} / \mathrm{h}$ (because of number of UEs out of ROI will increase with increase the speed of UEs) as clarified in Fig. 7. Additionally, the spectral efficiency depends on the channel conditions (CQI). CQI decreases to be reached a minimum values; therefore, the spectral efficiency becomes a minimum values as declared as [10]. The result of this relation, the spectral efficiency don't effect greatly with the high speed larger than $80 \mathrm{Km} / \mathrm{h}$.

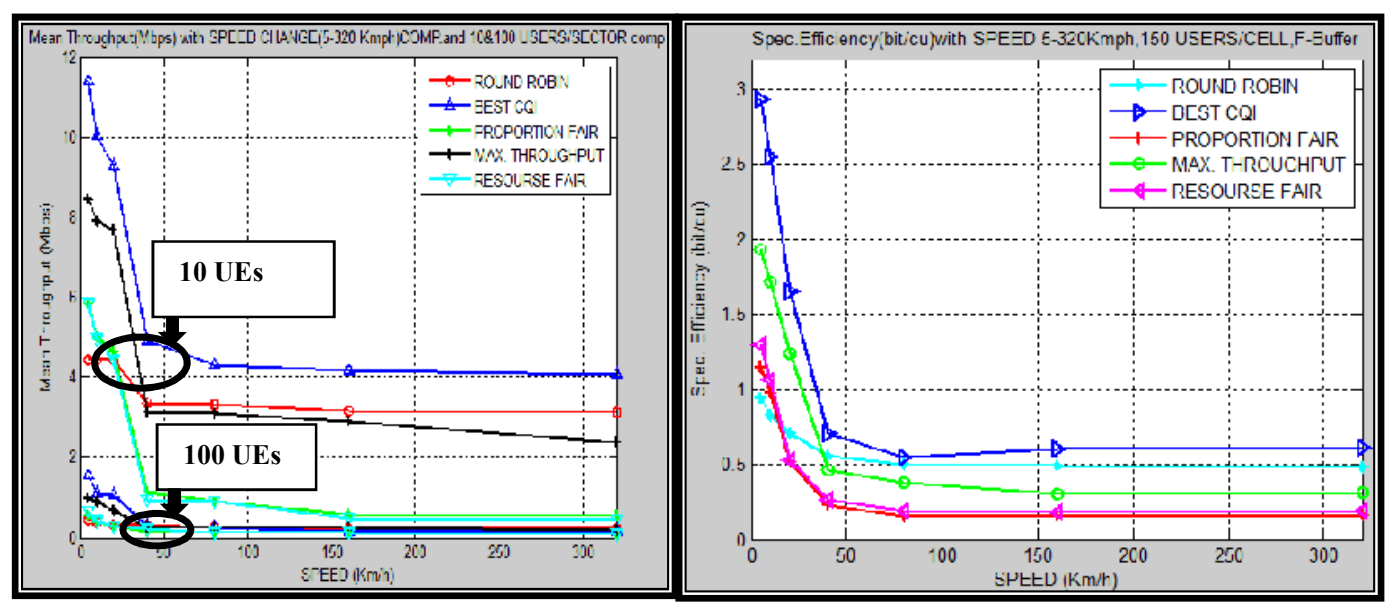

Fig. 18: Throughput vs. speed.

Fig. 19: Spectral efficiency vs. speed.

The resource fair achieves higher efficiency coefficient than the others when the speed is less than $10 \mathrm{Km} / \mathrm{h}$, the round robin has higher efficiency coefficient than the others when the speed of UEs is $80 \mathrm{Km} / \mathrm{h}$. On the other hand, Fig. 20 shows the efficiency coefficient of the best CQI with increasing the speed of UEs to achieve higher efficiency coefficient than the others when the speed of UEs is greater than $80 \mathrm{Km} / \mathrm{h}$ as illustrated by eq. 6 .

The effect of varying the speed of UEs on the fairness at 10UEs/eNB and the speed of UEs changes from $(5-320 \mathrm{Km} / \mathrm{h})$ is displayed in Fig. 21 . The resource fair has higher fairness than the others when the speed of UE is $10 \mathrm{Km} / \mathrm{h}$, while RR has higher fairness than the others when the speed of UE is greater than $10 \mathrm{Km} / \mathrm{h}$. 


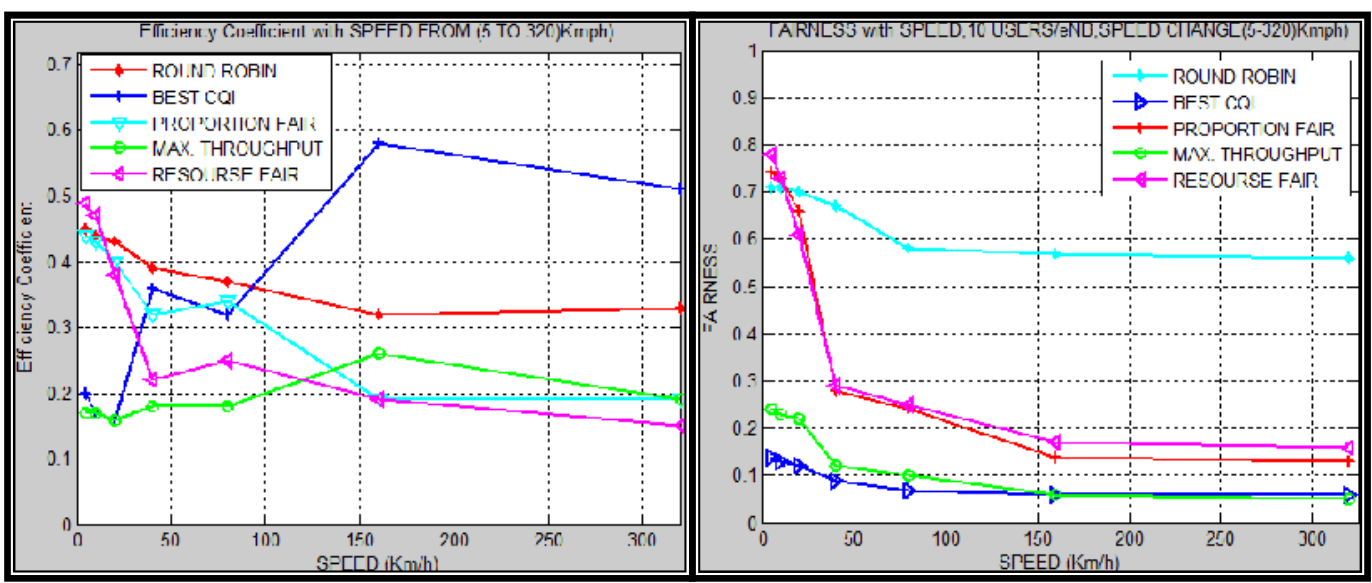

Fig. 20: Efficiency with speed.

Fig. 21: Fairness with the speed

\section{Conclusions and Recommendations}

As mentioned before, five types of downlink packet scheduling algorithms in LTE networks are simulated. It has been examined results of the impact of the throughput, the fairness, the efficiency coefficient and the spectral efficiency for each scheduling algorithm when the speed of UEs and the number of UEs have been changed. A new comparisons among them has been done through simulations executed of Vienna LTE system level Simulator v1.8 r1375.

The conclusions and recommendations from results are as following:

1- It is found that increasing the number of UEs and the speed of UEs will cause throughput degradation for all scheduling algorithms. It is obvious from the results that the Best CQI scheduling algorithm can achieve higher throughput of UEs, spectral efficiency than the others because of it is assigned a RBs to the UEs that have the high CQI; therefore, it provides lower fairness among UEs, also, it allocates all the resources to the nearest UEs whose have the best channel conditions as declared in equation (1). BCQI doesn't allocate any resources to the UEs at the edge of the cell.

2- When the speed of UEs exceeds $10 \mathrm{Km} / \mathrm{h}$, the best scheduling algorithm is round robin, it achieves a good efficiency, good Spectral Efficiency and higher fairness than the others and it is the unique scheduling algorithm which allocates resources to the UEs at the edge of the cell, as shown in Fig. 14 and Fig. 20.

3- The scheduling algorithms which are recommended to be used when the number of UEs are within the range (10-100)UEs/eNB at (5) Km/h speed of UEs, as shown in table 1.

4- The scheduling algorithms are recommended when the speed(S) of UEs are from 5 to $320 \mathrm{Km} / \mathrm{h}$ at (10)UEs/sector, as presented in table 2 . 
Table 1: The algorithms with no. of UEs from (10-100) UEs/eNB.

\begin{tabular}{|l|l|l|l|}
\hline \multicolumn{1}{|c|}{ Comparison criteria } & $(10-80)$ UEs/eNB & $(80-100)$ UEs/eNB & Figure no. \\
\hline Efficiency coefficient & Resource fair & Round robin & Fig. 13 \\
\hline $\begin{array}{l}\text { Throughput of UEs at a } \\
\text { cell-edge }\end{array}$ & Resource fair & Proportional fair & $\begin{array}{l}\text { Fig. 4, Fig. } \\
6 \text { and Fig. 9 }\end{array}$ \\
\hline Throughput of UEs & Best CQI & Best CQI & Fig. 10 \\
\hline Fairness & Resource fair & Resource fair & Fig. 11 \\
\hline Spectral Efficiency & Best CQI & Best CQI & Fig. 12 \\
\hline
\end{tabular}

Table 2: The algorithms with speed (S) of UEs from $(5-320) \mathrm{Km} / \mathrm{h}$.

\begin{tabular}{|l|l|l|l|l|}
\hline \multicolumn{1}{|c|}{$\begin{array}{c}\text { Comparison } \\
\text { criteria }\end{array}$} & $\mathrm{S} \leq 10 \mathrm{Km} / \mathrm{h}$ & $\begin{array}{l}\mathrm{S} \geq 10 \mathrm{Km} / \mathrm{h} \text { to } \\
\mathrm{S}=80 \mathrm{Km} / \mathrm{h}\end{array}$ & $\begin{array}{l}\mathrm{S} \geq 80 \mathrm{Km} / \mathrm{h} \\
\text { to } \\
\mathrm{S}=320 \mathrm{Km} / \mathrm{h}\end{array}$ & Figure no. \\
\hline $\begin{array}{l}\text { Efficiency } \\
\text { Coefficient }\end{array}$ & Resource fair & Round robin & Best CQI & Fig. 20 \\
\hline $\begin{array}{l}\text { throughput of UEs } \\
\text { at the edge of cell }\end{array}$ & $\begin{array}{l}\text { Proportional } \\
\text { fair }\end{array}$ & Round robin & Round robin & $\begin{array}{l}\text { Fig.15, Fig.16 } \\
\text { and Fig. 17 }\end{array}$ \\
\hline $\begin{array}{l}\text { Throughput } \\
\text { of UEs }\end{array}$ & Best CQI & Best CQI & Best CQI & Fig. 18 \\
\hline Fairness & Resource fair & Round robin & Round robin & Fig. 21 \\
\hline Spectral Efficiency & Best CQI & Best CQI & Best CQI & Fig. 19 \\
\hline
\end{tabular}

\section{References:}

[1] B. Liu, H. Tian, and Lingling Xu, “An Efficient Downlink Packet Scheduling Algorithm for Real Time Traffics in LTE Systems", The work is supported by the HUAWEI Project under No.YBWL2010221, IEEE, 2013.

[2] Capozzi, F.; Boggia, G.; Piro, G.; Grieco, L.A.; Camarda, P., "Downlink Packet Scheduling in LTE Cellular Networks: Key Design Issues and a Survey", Communications Surveys \& Tutorials, IEEE, vol. 15, no.2, pp.678-700, Second Quarter 2013.

[3] A. S. Sravani and K. Jagadeesh Babu, "Implementation of Scheduling Algorithms for LTE Downlink",International Journal of Advanced Electrical and Electronics Engineering (IJAEEE), ISSN (Print): 2278 - 8948, vol. 2, Issue -6, 2013.

[4] Dizhi Zhou; Wei Song; Baldo, N.; Miozzo, M., "Evaluation of TCP performance with LTE downlink schedulers in a vehicular Wireless environment" Communications and Mobile Computing Conference (IWCMC), 2013 9th International, vol., no., pp.10641069, 1-5 July 2013.

[5] R. Kwan, C. Leung and J. Zhang," Downlink Resource Scheduling in an LTE System", Mobile and Wireless Communications Physical Layer Development and Implementation, 2010. Available from: http://www.intechopen.com/books /mobile-and-wirelesscommunications-physical-layer-development-and implementatiom/downlink-resourcescheduling-in-an-lte-system 
[6] K. Ravindhra, S. S. Manohar, and U. S. Govindaswamy " Long Term Evolution Downlink Physical Layer Simulation in Matlab and Simulink" International Journal of Future Computer and Communication, Vol.1,No.2, August,2012.

[7] H. Al-Jaradat and K. Sandrasegaran, "On the Performance of PF, MLWDF and EXP/PF algorithms in LTE" Faculty of Engineering and Information Technology University of Technology Sydney, international journal of computers technology, June 15, 2013.

[8] S.A. Alqahtani and M. Alhassany "Comparing different LTE scheduling schemes," Wireless Communications and Mobile Computing Conference (IWCMC), 2013 9th International, vol., no., pp.264-269, 1-5 July 2013.

[9] Alqahtani, S.A.; Alhassany, M., "Performance Modeling and Evaluation of Novel Scheduling Algorithm for LTE Networks", Network Computing and Applications (NCA), 2013 12th IEEE International Symposium on, vol., no., pp.101,105, 22-24 August, 2013.

[10] M. H. Habaebi, J. Cheil, A.G. AL-Sakkaf and T. H. Dahawi " comparison between Scheduling techniques in LTE" International Islamic University Malaysia, Jalan Gombak 53100, Kuala Lumpur, Malaysia. IIUM Engineering Journal, Vol. 14, No. 1, 2013.

[11] F. Bendaoud, Marwen A. and Fedoua Didi, "Survey On Scheduling and Radio Resources Allocation In LTE", International Journal of Next-Generation Networks (IJNGN), DOI : 10.5121/ijngn.2014.6102Vol.6, No.1, March 2014.

[12] LTE System Level Simulator version v1.8 r1375 by Institute Of communication and Radio Frequency Engineering, Vienna University of Technology, Vienna, 2014. Web: http://www.nt.tuwien.ac.at/ltesimulator. 